\title{
A Malignant Mass in the Breast Is Not Always Breast Cancer
}

\author{
Fatih Selcukbiricik $^{\mathrm{a}}$ Deniz Tural ${ }^{\mathrm{a}} \quad$ Ayse Bay $^{\mathrm{a}}$ \\ Gulcin Sahingoz $^{\mathrm{a}}$ Sennur ilvan ${ }^{\mathrm{b}}$ Nil Molinas Mandel \\ Departments of ${ }^{\mathrm{a}} \mathrm{Clinical}$ Oncology and ${ }^{\mathrm{b}}$ Pathology, Cerrahpasa Medical Faculty, \\ University of Istanbul, Istanbul, Turkey
}

\section{Key Words}

Colon cancer · Breast metastasis · Chemotherapy · Biopsy

\begin{abstract}
A 37-year-old woman presented to the Internal Medicine Clinic with complaints of abdominal pain and constipation which had begun 3 months earlier. A colonoscopy was performed, and wall thickening of the sigmoid colon was detected. A biopsy of the sigmoid colon revealed a poorly differentiated, mucin-producing adenocarcinoma with a signet-ring pattern. No distant metastasis was detected. The patient was treated with chemotherapy consisting of 5-fluorouracil, leucovorin, and oxaliplatin. One and a half years later, a painless mass, which was not fixed to the skin, measuring $1 \mathrm{~cm}$ in diameter, was found in the lower outer quadrant of the left breast. A core biopsy of the mass was performed, and a histopathological report confirmed metastasis to the breast from mucinous adenocarcinoma of an intestinal primary.
\end{abstract}

\section{Introduction}

Colorectal cancer is the third most common cancer in the world. Cancer of the colon most commonly spreads to the lymph nodes, liver, lungs, and bones, in that order. Metastasis of colon cancer to the breast is quite rare. Although primary breast carcinoma is the most common tumor in women, even in patients with a history of cancer from other organs, metastases to the breast from solid tumors may be detected $[1,2]$. The most common primary tumor to metastasize to the breast is contralateral breast carcinoma. Every mass found in the breast should not inherently be regarded as primary breast cancer, and it should be kept in mind that, in a patient with a history of a solid tumor, the mass could be a metastasis of that solid tumor. Here, we report a rare case of aggressive colon cancer with metastasis to the breast, without liver or lung metastases. The patient was started on palliative chemotherapy with irinotecan, 5fluorouracil (5-FU), and leucovorin (FOLFOX4). Management of breast cancer metastasis is discussed below, accompanied by a review of the literature. 


\section{Case Report}

A 37-year-old woman presented to the Internal Medicine Clinic with complaints of abdominal pain and constipation which had begun 3 months earlier. Physical examination was unremarkable. A colonoscopy was performed, and wall thickening of the sigmoid colon was detected. A biopsy revealed poorly differentiated mucin-producing adenocarcinoma with a signet-ring pattern (fig. 1a). No distant metastasis was detected. After sigmoidectomy for T3N3M0 (stage III) adenocarcinoma of the colon, adjuvant FOLFOX4 was administered for six cycles on days 1 and 15, every two weeks.

One and a half years later, a painless mass, which was not fixed to the skin, measuring $1 \mathrm{~cm}$ in diameter, was found in the lower outer quadrant of the left breast. Mammography showed a welldefined nodule without microcalcifications and axillary lymphadenopathy, $1 \times 1 \mathrm{~cm}$ in size. Positron emission tomography showed a high FDG uptake in the left breast (fig. 2). In this clinical context, the suspicion that the patient's breast lump represented a metastatic deposit of her colon cancer was raised.

A core biopsy of the breast mass was performed. A histopathological study confirmed metastasis to the breast from a mucinous adenocarcinoma of gastrointestinal origin and a colonic-type pattern with signet-ring cells. Immunohistochemical results were negative for estrogen and progesterone receptors and GCDFP-15, and positive for cytokeratin 20 (CK-20) (fig. 1b). Surgical treatment was not preferred, and the patient was then started on palliative FOLFOX4 chemotherapy.

\section{Discussion}

Colorectal cancer is the third most common cancer in the world and second most common cause of cancer mortality after lung cancer. The risk for colorectal cancer increases with age, but $3 \%$ of colorectal cancers occur in patients younger than 40 years of age. Multiple factors like polyps, diet, inflammatory bowel disease, genetics, and smoking drive the transformation of healthy colorectal mucosa to cancer. Genetic and environmental factors, such as maintaining a low body mass index and exercising regularly, correlate with lower incidence rates. Metastases to the regional lymph nodes are found in $40-70 \%$ of cases at the time of resection, with metastases to more distant sites occurring most frequently in the liver, peritoneal cavity, and lung, followed by the adrenals, ovaries, and bone [3]. Metastatic spread of colonic tumors to the breast is rare and is usually in the context of widespread disease. When the disease is detected in its early, localized stages, the five-year survival rate is approximately $90 \%$. The prognosis for patients with metastatic disease is poor. In patients with distant metastases, the five-year survival rate is approximately 10\% [4]. Cancers of the colon, uterine corpus, breast and ovary frequently occur together, suggesting an underlying hormonal or dietary effect [5].

Primary breast cancer is the most common cancer afflicting women (incidence of $1 / 8-10$ ), and it is the second leading cause of cancer death overall (following lung cancer). Breast cancer which is detected early is curable, but it has a $10-20 \%$ chance of distant metastases occurring even 10-20 years after the initial diagnosis [6]. The most common sites of breast cancer metastasis are bone, lungs, and the liver [7]. Metastatic cancer to the breast is very rare $[8,9]$. The most common primary tumor is a contralateral breast carcinoma, followed by leukemia, melanoma, lymphoma, and ovary, lung, and gastric cancer [10].

Mammographic evaluation can be useful in the differential diagnosis of primary versus secondary breast cancer [10]. Metastasis to the breast from extramammary 
malignancies usually presents as rapidly growing mobile masses that are easily palpable but do not cause an overlying skin or nipple retraction [11]. The mammographic finding in such a case is a rounded and well-circumscribed mass. Typically, there is no spiculation, microcalcification or thickening of the skin [10]. As metastatic neoplasms can mimic benign or primary breast neoplasms clinically and radiologically, differential diagnosis between primary and metastatic breast neoplasms is not always easy. Because treatment can differ profoundly, accurate diagnosis is of paramount importance to avoid unnecessary radical surgical procedures. Excisional or incisional biopsy is the most commonly used procedure for diagnosis [6].

In some equivocal cases, immunohistochemistry can help to make an accurate diagnosis [1]. The great majority of breast tumors are CK7 positive and CK20 negative, while colorectal carcinomas are usually CK7 negative and CK20 positive. When a breast tumor is negative for all breast markers (ER, PR, Her2, GCDP15, BCA, and CK7) and positive for CK20 and CDX2, this identifies the tumor as being colon cancer metastatic to the breast [4]. If the patient is found to have a tumor of breast origin, treatment would include local excision, radiotherapy, and, if the tumor is receptor positive, systemic hormonal manipulation [4].

There are 20 cases of colon cancer metastatic to the breast in the literature. The real incidence is difficult to estimate and is probably much higher [12]. Typically, these cases are associated with disseminated metastatic disease and imply a poor prognosis $[10,13]$. Mastectomy in this setting, other than palliation, has a limited role. The main form of treatment for these patients is systemic chemotherapy [7].

In conclusion, colon cancer metastatic to the breast is very rare. Metastasis to the breast usually indicates disseminated disease, and the prognosis is poor. Mastectomy is controversial, and standard systemic treatment should be applied. 

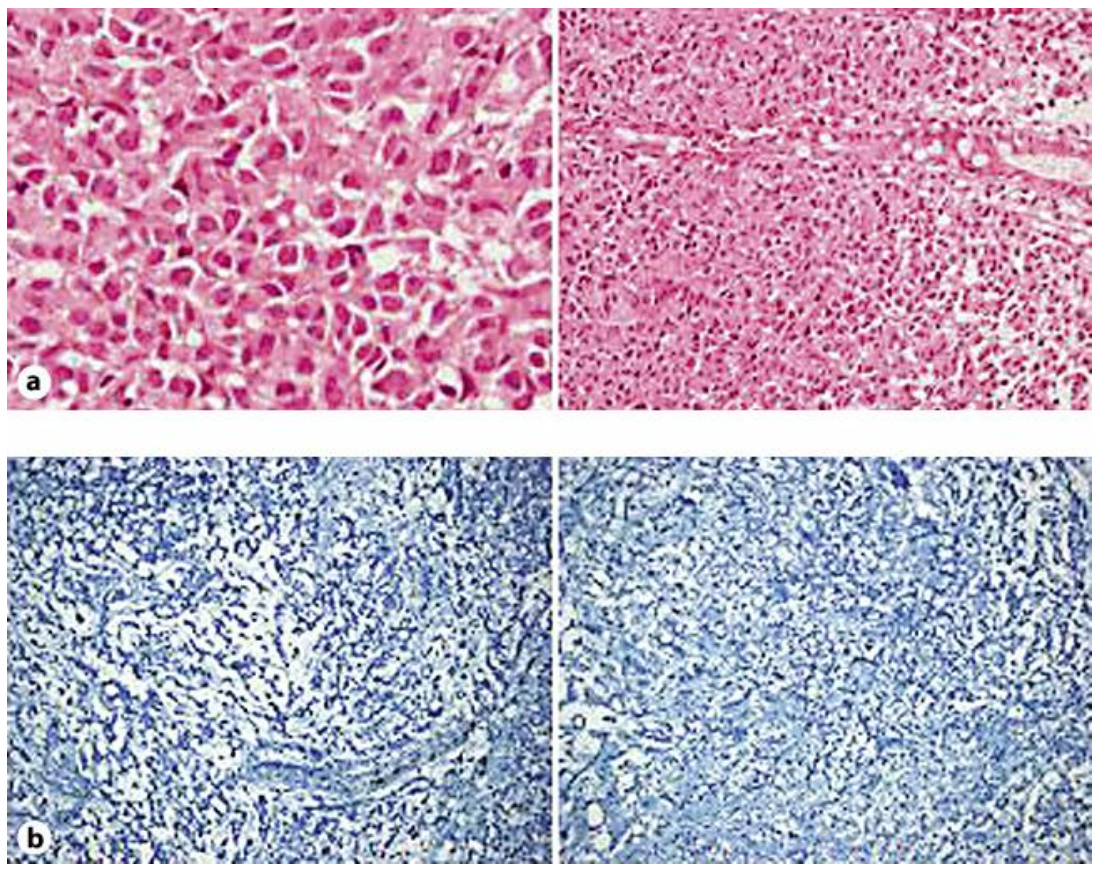

Fig. 1. a Sigmoid colon: signet-ring pattern component, poorly differentiated adenocarcinoma with an extracellular mucin-producing component, invasion of submucosal blood vessels $(\times 400)$. b Trucut biopsy of the left breast: poorly differentiated adenocarcinoma with extracellular mucin and a focal signet-ring component, estrogen and progesterone negative $(E R \times 100, P R \times 200)$.
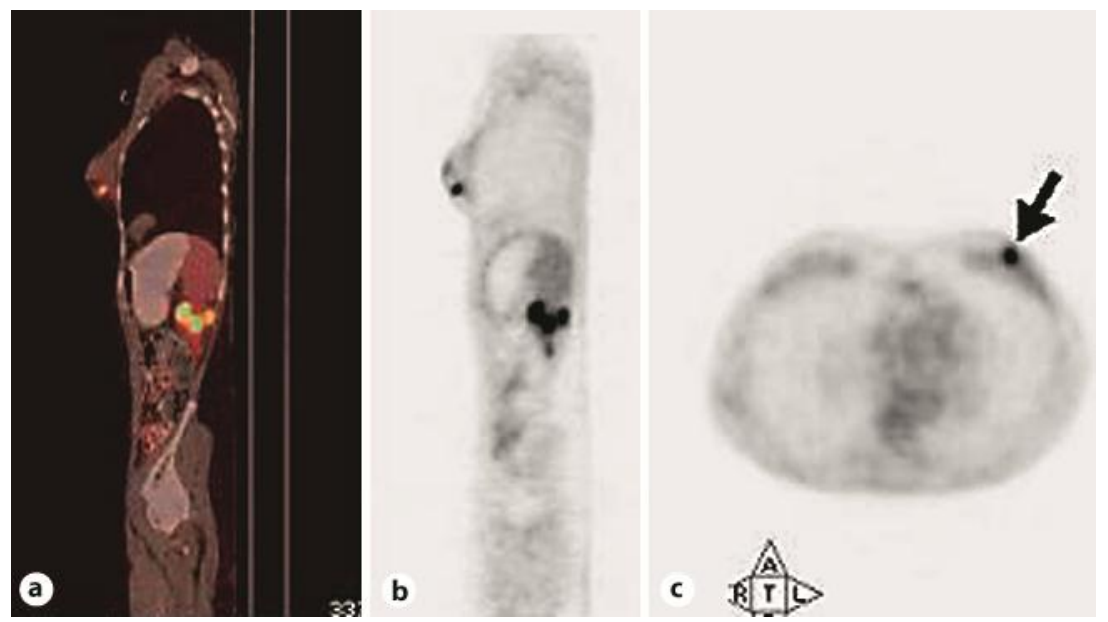

Fig. 2. A 37-year-old woman with a history of sigmoid colon cancer underwent FDG PET/CT imaging for restaging of the primary disease. Therefore she was intravenously injected $475 \mathrm{MBq}(12.8 \mathrm{mCi})$ of 18F-FDG after a 6-hour fasting period. After $1 \mathrm{~h}$ of waiting time in a silent room, the patient was imaged using an integrated PET/CT camera, which consists of a 6-slice CT gantry integrated in a LSObased fullring PET scanner (Siemens Biograph 6, Ill., USA). FDG PET/CT images showed a hypermetabolic lesion in the left breast consistent with malign lesions. 


\section{References}

1 Bhirangi KS, Pinto DJDT, Crosbie JJ: Solitary breast metastasis from carcinoma of colon. Ulster Med 1997;66:138-139.

-2 Ozakyol A, Isiksoy S: Breast metastasis of primary colon cancer. Am J Gastroenterol 2000;95:2149.

-3 Sanchez LD, Chelliah T: Rare case of breast tumor secondary to rectal adenocarcinoma. Southern Med Association 2008;28:1062-1064.

$>4$ de Bobadilla FL, Villanueva GA, Collado M, et al: Breast metastasis of primary colon cancer. Rev Esp Enferm Dig 2004;96:415-419.

5 Ho YY, Wai-Kit L: Metastasis to the breast from an adenocarcinoma of colon. J Clin Ultrasound 2009;37:239-241.

6 Oksuzoglu B, Abali H, Guler N, et al: Metastasis to the breast from nonmammarian solid neoplasms: a report of five cases. Med Oncol 2003;20:295-300.

7 Li HC, Patel P, Kapur P: Metastatic rectal cancer to the breast. Rare Tumors 2009;1:e22.

$>8$ Barthelmes L, Simpson JS, et al: Metastasis of primary colon cancer to the breast. Breast Care 2010;5:2325.

-9 Boice JD Jr, Curtis RE, et al: Multiple primary cancers in Connecticut, 1935-82. Yale J Biol Med 1986;59:533-545.

10 Mihai R, Christe-Brown J, Bristol J: Breast metastases from colorectal carcinoma. Breast 2004;13:155158.

11 Michalopoulos A, Pepdopoulos V, et al: Metastatic breast adenocarcinoma masquerading as colonic primary. Tech Coloproctol 2004;8:135-137.

12 Prete CD, Babkowski RC, et al: Breast metastasis from colonic primary. Connecticut Med 2009;73:399401.

13 Alberts SR, Goldberg RM: Gastrointestinal tract cancers; in Casciato DA, Territo MC (eds): Manual of Clinical Oncology. Philadelphia, Lippincott Williams \& Wilkins, 2009, pp 188-237. 